\title{
Salvage lymph node dissection for prostate-specific membrane antigen (PSMA) positron emission tomography (PET)-identified oligometastatic disease
}

Adam Bobrowski ${ }^{1}$; Ur Metser ${ }^{2}$; Antonio Finelli ${ }^{3}$; Neil Fleshner ${ }^{3}$; Alejandro Berlin ${ }^{4}$; Nathan Perlis $^{3}$; Girish S. Kulkarni ${ }^{3}$; Peter Chung ${ }^{4}$; Kopika Kuhathaas ${ }^{3}$; Eshetu G. Atenafu ${ }^{5}$; Robert J. Hamilton $^{3}$

${ }^{1}$ Faculty of Medicine, University of Toronto, Toronto, ON, Canada; ${ }^{2}$ Department of Diagnostic Radiology, Princess Margaret Cancer Centre, Toronto, ON, Canada; ${ }^{3}$ Division of Urology, Princess Margaret Cancer Centre, Toronto, ON, Canada; ${ }^{4}$ Department of Radiation Oncology, Princess Margaret Cancer Centre, Toronto, ON, Canada; ${ }^{5}$ Department of Biostatistics, Princess Margaret Cancer Centre, Toronto, ON, Canada

Cite as: Bobrowski A, Metser U, Finelli A, et al. Salvage lymph node dissection for prostatespecific membrane antigen (PSMA) positron emission tomography (PET)-identified oligometastatic disease. Can Urol Assoc J 2021 March 22; Epub ahead of print. http://dx.doi.org/10.5489/cuaj.6988

Published online March 22, 2021

$* * *$

\section{Abstract}

Introduction: The availability of prostate-specific membrane antigen (PSMA) positron emission tomography (PET)/computed tomography (CT) imaging, particularly in the setting of rising prostate-specific antigen (PSA) after definitive treatment, has led to oligometastatic prostate cancer being increasingly identified. Despite the enthusiasm surrounding treating oligometastatic disease, it has been relatively understudied. We sought to review our salvage lymphadenectomy experience in the PSMA PET/CT era.

Methods: We retrospectively reviewed patients undergoing lymphadenectomy following curative intent primary therapy with rising PSA who had undergone a PSMA PET/CT identifying oligometastatic disease (defined as $\leq 5$ PSMA-avid lesions) between January 2016 to April 2020. The primary endpoint was complete response, defined as achieving a PSA $<0.2$ $\mathrm{ng} / \mathrm{ml}$ without concomitant androgen deprivation therapy (ADT).

Results: Twenty-two patients were included. Primary curative therapy included radical prostatectomy (86.4\%) and brachytherapy (13.6\%). Median PSA at salvage surgery was 1.72 $\mathrm{ng} / \mathrm{ml}$. Pelvic lymph node dissection was the most performed procedure $(72.7 \%)$. Median node yield was 10.5 , with a median of 1.5 positive nodes on pathology. Eight patients $(36.4 \%)$ achieved PSA $<0.2$, with six (27.3\%) remaining with PSA $<0.2$ after a median followup of 23.1 months. Nine $(40.9 \%)$ had an initial PSA decline, but nadired $\geq 0.2$, and in five $(22.7 \%)$ the PSA 
rose immediately after surgery. Overall, ADT was commenced in seven patients $(31.8 \%)$ at a median of 10.1 months post-salvage surgery.

Conclusions: In our series of salvage dissection for PSMA-PET-detected nodal oligometastases, approximately a third achieved PSA $<0.2$; yet, it was only durable in $27 \%$. Prospective trials of salvage nodal radiation are ongoing; however, more prospective trials of salvage node dissection are needed.

\section{Introduction}

Between $27 \%-53 \%$ of localized prostate cancers (PCa) treated with radical prostatectomy (RP) or radiation thearpy (RT) will experience biochemical recurrence (BCR). ${ }^{3-6}$ Approximately $30 \%{ }^{-}$ $40 \%$ of patients with BCR will undergo clinical progression at long-term follow-up. ${ }^{7}$

Predominantly, these patients are managed with immediate or delayed androgen deprivation therapy (ADT) regardless of the location or extent of recurrence. 8,9

Hellman and Weichselbaum, in their seminal paper on oligometastatic disease, challenged the dogma that cancer exists in a dichotomous state of local or systemic disease ${ }^{10}$ Rather, they propose the oligometastatic state acts as a transitional intermediary where lesions have yet achieved their full metastatic potential and manifest a range of clinical possibilities. ${ }^{11}$

Targeting oligometastatic PCa with metastases-directed therapy (MDT) has become a topic of interest. State-of-the-art imaging modalities such as the prostate-specific membrane antigen (PSMA) positron emission tomography (PET)/computerized tomography (CT) and PSMA $\mathrm{PET} / \mathrm{magnetic}$ resonance imaging (MRI) has demonstrated high diagnostic accuracy in identifying low-volume PCa in biochemical recurrence patients. ${ }^{11-13}$ In this context, salvage lymph node dissection (SLND) carries promise of an individualized approach to prolonging progression-free survival (PFS), delaying time to palliative ADT, and potentially prolonging survival. ${ }^{13}$ Multiple retrospective studies have assessed the role of SLND in this setting with encouraging results; however, there is a lack of level 1 evidence to define the role and value of SLND in oligometastatic PCa. ${ }^{14,15}$ Further understanding of the role of surgery in the MDT sphere remains an important pre-requisite in allowing clinicians to exercise better judgement when deriving therapeutic approaches to this pathology.

The aim of the present study was to evaluate the oncologic efficacy and safety of SLND in patients found to have oligometastatic PCa on PSMA-PET/CT imaging at the Princess Margaret Cancer Centre. 


\section{Methods}

\section{Patient selection}

Following approval from the institutional Research Ethics Board, the electronic patient record and the institutional Operating Room Scheduling System were employed to retrospectively identify patients with a rising PSA following curative intent primary therapy who had undergone SLND for PSMA PET/CT identified oligometastatic PCa (defined as $\leq 5$ PSMA-avid lesions) between January 2016 to April 2020. Inclusion eligibility was dependent on the following criteria: histologically proven diagnosis of PCa, BCR (defined as PSA level $\geq 0.2 \mathrm{ng} / \mathrm{ml}$ following RP or PSA nadir plus $\geq 2 \mathrm{ng} / \mathrm{ml}$ following RT) after curative intent primary therapy (RP, RT, brachytherapy, ADT, or a combination) and detection of $\leq 5$ PSMA-avid lesions. ${ }^{17}$ All RP procedures included simultaneous pelvic lymph node dissections. Patients were permitted if they had recurrence after initial primary therapy and received salvage therapy (e.g. salvage RT +/- ADT, salvage RP), but at the time of enrollment had rising PSA with non-castrate testosterone levels and off ADT. Subjects included in this retrospective chart review elected to undergo PSMA PET/CT imaging and were provided with options for treatment of their oligometastatic disease, such as RT and ADT, prior to agreeing to surgical intervention. Ultimately, patients who elected to undergo another mode of therapy other than SLND were not included in the study. Patients were also excluded in the case of M1b or M1c lesions, identification of lesions by a modality other than PSMA-PET/CT imaging, or patients with $<90$ days of post-operative follow-up. Since PSMA-PET/CT scans were unavailable at our institution until 2017, six patients (27.3\%) underwent ${ }^{68}$ Ga-PSMA PET/CT from external institutions. The remainder of the cohort $(n=16,72.7 \%)$ underwent ${ }^{18} \mathrm{~F}$-DCFPyL PSMA PET/CT scans at our institution. Patients who had undergone a PSMA PET/CT identifying oligometastatic disease, as reviewed by both the reporting radiologist and treating urologist, were considered for surgery. Operations of interest included pelvic lymph node dissection (PLND), and retroperitoneal lymph node dissection (RPLND). Both open and robotic procedures were considered.

\section{Surgical outcomes and followup}

Patients were staged with either ${ }^{68} \mathrm{Ga}-\mathrm{PSMA}$ PET/CT or ${ }^{18} \mathrm{~F}$-DCFPyL PET/CT and subsequently underwent SLND. All procedures and pathological assessments were conducted at our institution. The surgical dissection templates were targeted to PSMA-avid lesions visualized on PSMA PET/CT imaging. The PLND was performed when PSMA PET/CT showed positive spots in the pelvic region. Unilateral or bilateral dissections were conducted depending on the laterality of PSMA uptake. Patients received RPLND when PSMA PET/CT showed positive spots in the retroperitoneum. A combination of procedures was performed if PSMA-avid nodes were visualized in both anatomic locations. The method of targeted templates was employed to maximize therapeutic benefit, while minimizing the risk of complication to ensure patient safety. 
Surgical, pathological and post-operative variables were collected to characterize outcomes. Surgical end points included: operative time, estimated blood loss, intra-operative transfusions, length of hospital stay, and complications (measured via the Clavien-Dindo classification system). Pathological end points included: number and histologic positivity of resected nodes, surgical margins, and extranodal extension. Post-operative outcomes were assessed during regular follow-up. PSA rise was defined as PSA $\geq 0.2 \mathrm{ng} / \mathrm{ml}$ for patients with a nadir $<0.2 \mathrm{ng} / \mathrm{ml}$ or two sequential increases if PSA nadired $\geq 0.2 \mathrm{ng} / \mathrm{ml}$ ). Subsequent salvage therapy was also recorded.

The primary end-point was "complete response", defined as achieving a PSA $<0.2 \mathrm{ng} / \mathrm{ml}$ without concomitant ADT. Secondary end-points included ADT-free survival (ADT-FS), metastasis-free survival (MFS), and progression-free Survival (PFS). MFS was defined as time from salvage node dissection to development of any new lesions detected on conventional or PET imaging. PFS was defined as time from salvage node dissection to post-operative PSA rise, new lesions on imaging, or initiation of treatment. Disease-specific survival and overall survival were not assessed, as no patients died during follow-up.

\section{Statistical analysis}

Categorical variables were summarized using counts and percentages, while continuous variables were represented as medians, and ranges. The Kaplan-Meier product-limit method was employed to calculate rates of ADT-FS, MFS, and PFS. The percentage change in PSA from pre-operative level to the post-operative PSA nadir was reported for each patient using a waterfall plot. A Chisquared test was used to assess differences in PSA response to salvage therapy, as well as discordance between pathologic and radiologic findings. Statistical analyses were performed using version 9.4 of the SAS system for Windows (2002-2012 SAS Institute, Cary, NC, USA). An independent biostatistician was used to reduce confirmation bias.

\section{Results}

\section{Cohort characteristics}

Twenty-two patients with BCR PCa following primary curative therapy were found to have oligometastatic nodal lesions on PSMA PET/CT imaging and underwent SLND. Patients were followed for a median of 23.1 months following salvage surgery. Table 1 summarizes the patient and disease characteristics of this cohort.

The most common modality of primary treatment was RP (81.8\%). RT was employed in $72.7 \%$ following PSA rise after RP at a median of 24 months and five patients $(22.7 \%)$ were placed on intermittent-intent ADT after their RP for a median of 9.67 months. However, none of the 22 patients in this cohort were on ADT at the time of PSMA-PET or SLND, with a median time off ADT of 49.7 months. Moreover, none had castrate testosterone levels at the time of SLND. 
The median interval from PCa diagnosis to oligometastatic lesions on PSMA PET/CT imaging was 5.80 years.

\section{Oncologic outcomes of SLND}

Operative and pathological outcome data are summarized in Table 2 and Table 3 , respectively. There was a median of 2 lesions on imaging at a median PSA of $1.50 \mathrm{ng} / \mathrm{ml}$ (Table 2). To treat oligometastatic lesions, 16 patients (72.7\%) underwent PLND (12 unilateral and 4 bilateral), 2 patients underwent RPLND, and 4 patients (18.2\%) underwent both a PLND and RPLND. Two patients underwent prostate-directed surgery at the time of SLND, consisting of RP and photodynamic therapy. The median node yield was 10.5 with a median of 1.5 positive nodes on histopathology.

Patterns of first progression following salvage surgery were recorded. The percentage change in PSA from pre-operative levels to post-operative nadir is shown in Figure 1. Eight (36.4\%) achieved a PSA of $<0.2 \mathrm{ng} / \mathrm{ml}$, with 6 of these patients $(27.2 \%)$ remaining with a PSA of $<0.2$ after a median follow-up of 23.1 months. Nine (40.9\%) patients had an initial PSA decline, but nadired $\geq 0.2$, and another 5 patients $(22.7 \%)$ experienced a rise in PSA immediately after surgery. A comparison of patients with PSA decline (36.4\%) and PSA rise/constant (63.6\%) following SLND was assessed utilizing variables including, mode of primary treatment, lymph node dissection at the time of primary treatment, previous ADT use, duration of ADT use, and time from primary treatment to PSA rise. No statistically significant differences were appreciated (Supplementary Table 1).

Three patients (13.6\%) did not have any identifiable cancer in their resected nodes. In one such patient, the PSA persistently rose immediately after surgery; however, in the other 2 , the PSA declined but nadired at $>0.2$. To assess this discordance further, a comparison of patients with discordant pathologic and radiologic results was conducted. Comparators including number of pathologically-proven nodes removed location of metastasis on PSMA PET/CT, type and extent of SLND, and mode of primary treatment were not found to be significantly different between the two cohorts (Supplementary Table 2).

The median PFS for the cohort was 5.69 months (95\% confidence interval (CI), 3.1610.6) (Figure 2). Median MFS estimate was 46.4 months ( $95 \% \mathrm{CI})$. At final follow-up, 9 patients $(40.9 \%)$ were found to have recurrence on imaging at median of 4.44 months (Figure 3$)$. Five recurrences were found using PSMA PET/CT imaging, while 4 patients had visible lesions on follow-up CT abdomen and pelvis (Table 2). The majority of recurrences were nodal and confined to the pelvic and retroperitoneal lymph nodes. One patient registered multiple liver metastasis in addition to pelvic and retroperitoneal nodal recurrences on $\mathrm{CT}$, and one patient was found to have a recurrence of small cell PCa at the bulbar urethra on cystoscopy.

Following the establishment of disease progression, 10 patients underwent subsequent therapy (Table 3). Seven patients were initiated on ADT at a median of 10.1 months following SLND. The median ADT-FS was not reached during the follow-up period; however, the 1-year 
and 2-year ADT-FS post-SLND was $80.6 \%$ and 68.2\%, respectively (95\% CI, 56.1-92.3) (Figure 4). Four patients underwent RT at a median of 6.0 months and one patient was placed on chemotherapy at 5.7 months post-operatively.

\section{Safety of SLND}

Four patients experienced post-operative complications. Grade I and II complications were experienced by one patient $(4.76 \%)$ respectively. These complications consisted of wound packing for partial dehiscence of the incision site, and the management of a large hematoma. Two patients (9.52\%) experienced grade IIIb complications: one ureteric injury requiring intraoperative repair; one iliac vein injury requiring repair and blood transfusion. In the postoperative period, this latter patient developed a deep vein thrombosis necessitating anticoagulation and the placement of an inferior vena cava filter. The remainder of operative safety data is depicted in Table 4.

\section{Discussion}

The management of oligometastatic PCa continues to be a research priority for cancer care providers. SLND represents a treatment option in the urologic oncologist's armamentarium that theoretically could provide survival benefit and delay to ADT initiation in patients with nodal recurrence. However, only retrospective studies are available for the evaluation of SLND. A recent systematic review highlighted that most published series are composed of small and heterogeneous cohorts, utilizing mostly choline-based PET imaging, making it challenging to implement contemporary treatment recommendations. ${ }^{18}$ While the European Association of Urology guidelines have considered SLND to be a potential treatment for patients with nodal recurrent $\mathrm{PCa}$, the Canadian Urological Association guidelines have yet to endorse SLND as a means of managing oligometastatisis. ${ }^{19-21}$

In our study of SLND performed via a targeted template in PSMA-PET/CT identified oligometastatic disease at Princess Margaret Cancer Centre, we observed over a third (36.4\%) achieved PSA $<0.2$, with just over a quarter $(27.2 \%)$ of the cohort remaining with PSA $<0.2$ after a median follow-up of 23.1 months for the whole cohort. ADT was commenced in seven patients (31.8\%) at a median of 10.1 months post-salvage surgery. While our findings demonstrated that the type of primary therapy and previous ADT use did not impact PSA response post-SLND, the results were not statistically significant.

The introduction of molecular imaging techniques like the PSMA PET/CT has allowed clinicians to re-stage patients with recurrent PCa even at very low PSA levels. ${ }^{22}$ Prior to PSMAbased tracers being available, choline-PET was utilized and thus comprises the majority of studies of SLND to date. ${ }^{23-33}$ In our study, we only report on patients who had oligometastatic disease identified on PSMA PET (both ${ }^{68} \mathrm{Ga}$-PSMA and ${ }^{18} \mathrm{~F}$-DCFPyL PSMA PET/CT). Since PSMA-PET/CT scans were unavailable at our institution until 2017, six patients $(27.3 \%)$ underwent ${ }^{68} \mathrm{Ga}$-PSMA PET/CT from external institutions while the remainder of the cohort 
$(\mathrm{n}=16,72.7 \%)$ underwent ${ }^{18} \mathrm{~F}$-DCFPyL PSMA PET/CT scans at our institution. Further, the majority (72.7\%) of our cohort was imaged using ${ }^{18} \mathrm{~F}$-DCFPyL PSMA PET/CT imaging. Studies suggest ${ }^{18} \mathrm{~F}$-DCFPyL may have better performance characteristics than ${ }^{68} \mathrm{Ga}$-PSMA PET/CT in the BCR setting. ${ }^{34}$ For these reasons, our study represents a unique contribution to the retrospective literature on SLND.

After SLND, $36.4 \%$ of patients achieved a PSA $<0.2 \mathrm{ng} / \mathrm{ml}$ with another $40.9 \%$ declining, but nadiring $\geq 0.2$. While this proportion of patients with a meaningful PSA response is consistent with other series, our estimated PFS was shorter at 5.69 months than seen in other series. ${ }^{18,23-33,35}$ It is important to note that other studies had larger proportions of patients who were on ADT at the time of SLND, or who received adjuvant ADT after SLND. ${ }^{15,18,23-32}$ This likely explains the longer PFS. The lasting impact of ADT on testosterone recovery and the potential upregulation of PSMA expression in the setting of ADT may be contributory to these results. ${ }^{36-38}$

We did observe cases where the pathology was in disagreement with the PSMA PET/CT scan. In three patients who underwent a unilateral pelvic lymph node dissection, no positive lymph nodes were retrieved. One such patient demonstrated a post-operative rise in PSA with negative CT imaging. A repeat PSMA PET/CT was conducted in one of these patients demonstrating that the PSMA-avid nodes were missed in the operative field. The PSA of the other two patients nadired above 0.2 and missed nodes from one patient being visible on CT imaging. Discrepancies between pre-operative PSMA PET/CT imaging findings and histopathologic outcomes following SLND have been described in previous studies. ${ }^{32,33,39}$ The often-challenging location of recurrent small PCa lesions and fibrotic changes associated with primary surgery and adjuvant radiotherapy compound the difficulty of these complicated procedures. Advances in radiolabeling of PSMA ligands with ${ }^{111}$ Indium and ${ }^{99 \mathrm{~m}}$ Technetium as $\gamma$ emitters may enable for improved intraoperative identification and removal of metastatic lesions when used in tandem with PET/CT imaging. ${ }^{40,41}$ Nonetheless, the outcomes of PSMA radioligand surgery need to be further defined.

For the purposes of this study, ADT-FS was selected as an endpoint as it is clinically relevant to avoid premature and unnecessary initiation of ADT. This systemic therapy has a significant impact patients from both a health-related quality of life and healthcare expenditures standpoint. ${ }^{14,42}$ While the median estimate of ADT-FS was not reached during the follow-up period, the 1-year and 2-year ADT-FS post-SLND was $80.6 \%$ and $68.2 \%$, respectively. This finding is similar to the phase II STOMP trial results, which reported that MDT (composed of surgery or stereotactic body RT) significantly improved ADT-FS compared to surveillance (21 months vs. 13 months, $\mathrm{P}=0.11) .{ }^{43}$ Other studies, albeit of retrospective design, have observed a similar time off ADT of around 2 years following MDT. ${ }^{15,18}$ Despite this convergence of data, more prospective studies are needed to hone the estimate of the average delay in ADT with SLND to better counsel our patients. 
The morbidity associated with SLND is not trivial. Approximately $18 \%$ of patients encountered surgery-related complications. Fortunately, only two patients experienced ClavienDindo Grade III complications. Our complication rate and proportion of high-grade complications were similar to other series. ${ }^{17}$ The most frequent complications based on the literature include lymphorrhagia and lymphocele drainage (weighted average of $7.7 \%$ and $4.9 \%$, respectively), neither of which occurred in our study. ${ }^{18,24-32,36}$ The weighted average of surgical re-intervention is approximately $1.2 \% .^{23-31,35}$ Anatomical alterations from previous PCa treatments may play a role in SLND complications.

Our study has some limitations. It is retrospective, and single-armed. It represents a highly selected group of patients based on multiple factors, including patient choice. As such the patient population is heterogeneous based on primary therapy, salvage treatments, nodal recurrence patterns and mode of nodal dissection. The accessibility of PET/CT imaging in Ontario was limited until recently resulting in patients seeking this resource from external institutions. As a result, patients were imaged using different PSMA tracers. A further limitation is that a repeat PET/CT was not conducted for all patients that did not respond biochemically to surgery to determine removal of PSMA-avid nodes. In cases where this was done, the PSMAavid node was only a few millimeters in size and challenging to extract. To our knowledge this study is the first Canadian report on the use of SLND in the treatment of oligometastatic PCa lesions found on PSMA PET/CT. As such, the results of this study can act as a springboard for future trials.

\section{Conclusions}

While there is a rationale for managing oligometastatic PCa with salvage therapy, it is important for clinicians to communicate realistic expectations to patients. Only about a third of our cohort experienced a meaningful PSA response, and this effect was only durable in $27 \%$ of cases. While SLND may prolong ADT initiation, the likelihood for cure is low and the complication rate of these procedures is not insignificant. Long-term survival impact of surgically resecting oligometastatic disease remains unknown. Prospective trials of salvage nodal radiation are ongoing with sizeable numbers and meaningful survival endpoints. Prospective trials of SLND, in contrast, are few in number and size and, as a result, further trials are encouraged and necessary. For now, SLND remains an experimental treatment. 


\section{References}

1. Fradet Y, Klotz L, Trachtenberg J, et al. The burden of prostate cancer in Canada. Can Urol Assoc J 2009 Jun;3:S92.

2. Canadian Cancer Society. Release notice-Canadian Cancer Statistics 2019. Health promotion and chronic disease prevention in Canada: research, policy and practice. 2019;8:255.

3. Statistics Advisory Committee. Canadian cancer statistics 2019. Toronto: Canadian Cancer Society; 2019.

4. Eggener SE, Scardino PT, Walsh PC, et al. Predicting 15-year prostate cancer specific mortality after radical prostatectomy. $J$ Urol 2011;3:869-75.

5. D'Amico AV, Moul J, Carroll PR, et al. Cancer-specific mortality after surgery or radiation for patients with clinically localized prostate cancer managed during the prostate-specific antigen era. J Clin Oncol 2003;11:2163-72.

6. Hamdy FC, Donovan JL, Lane J, Mason M, Metcalfe C, Holding P, Davis M, Peters TJ, Turner EL, Martin RM, Oxley J. 10-year outcomes after monitoring, surgery, or radiotherapy for localized prostate cancer. $N$ Engl J Med 2016;375:1415-24.

7. Artibani W, Porcaro AB, De Marco V, et al. Management of biochemical recurrence after primary curative treatment for prostate cancer: a review. Urol Int 2018;3:251-62.

8. Boorjian SA, Thompson RH, Tollefson MK, et al. Long-term risk of clinical progression after biochemical recurrence following radical prostatectomy: the impact of time from surgery to recurrence. Eur Urol 2011;6:893-9.

9. Cornford P, Bellmunt J, Bolla M, et al. EAU-ESTRO-SIOG guidelines on prostate cancer. Part II: treatment of relapsing, metastatic, and castration-resistant prostate cancer. Eur Urol 2017;4:630-42.

10. De Bruycker A, Lambert B, Claeys T, et al. Prevalence and prognosis of low-volume, oligorecurrent, hormone-sensitive prostate cancer amenable to lesion ablative therapy. BJUI 2017;6:815-21.

11. Weichselbaum RR, Hellman S. Oligometastases revisited. Nat Rev Clin Oncol 2011;86:378.

12. Tosoian JJ, Gorin MA, Ross AE, et al. Oligometastatic prostate cancer: definitions, clinical outcomes, and treatment considerations. Nat Rev Urol 2017;1:15.

13. Pawson A, Ghumman Z, Kuo PH, et al. A review of prostate cancer imaging, positron emission tomography, and radiopharmaceutical-based therapy. Can Urol Assoc J 2020;4:130.

14. Tosoian JJ, Gorin MA, Ross AE, et al. Oligometastatic prostate cancer: definitions, clinical outcomes, and treatment considerations Nat Rev Urol 2017;14:15.

15. Khoo V. New concepts in prostate cancer management: the conundrum of managing oligometastatic disease in prostate cancer-through the looking glass darkly. Clinical radiology. 2019;11:865-75.

16. Heidenreich A, Moul JW, Shariat S, et al. Role of salvage lymph node dissection in prostate cancer. Curr Opin Urol 2016;6:581-9.

17. Ost P, Bossi A, Decaestecker K, et al. Metastasis-directed therapy of regional and distant recurrences after curative treatment of prostate cancer: a systematic review of the literature. Eur Urol 2015;5:852-63. 
18. Danielson B, Saad F, So A, et al. Management algorithms for prostate-specific antigen progression in prostate cancer: Biochemical recurrence after definitive therapy and progression to non-metastatic castrate-resistant prostate cancer. Can Urol Assoc J 2019;12:420.

19. Ploussard G, Gandaglia G, Borgmann H, et al. Salvage lymph node dissection for nodal recurrent prostate cancer: a systematic review. Eur Urol 2019;4:493-504.

20. Mottet N, Bellmunt J, Bolla M, et al. EAU-ESTRO-SIOG guidelines on prostate cancer. Part 1: screening, diagnosis, and local treatment with curative intent. Eur Urol 2017;4:618-29.

21. Saad F, Aprikian A, Finelli A, et al. 2019 Canadian Urological Association (CUA)Canadian Uro Oncology Group (CUOG) guideline: Management of castration-resistant prostate cancer (CRPC). Can Urol Assoc J 2019;10:307.

22. So A, Chi K, Danielson B, et al. Canadian Urological Association-Canadian Urologic Oncology Group guideline on metastatic castration-naive and castration-sensitive prostate cancer. Can Urol Assoc J 2020;2:17.

23. De Bruycker A, De Bleser E, Decaestecker K, et al. Nodal oligorecurrent prostate cancer: Anatomic pattern of possible treatment failure in relation to elective surgical and radiotherapy treatment templates. Eur Urol 2019;5:826-33.

24. Kimura S, Abufaraj M, Janisch F, et al. Performance of [68 Ga] Ga-PSMA 11 PET for detecting prostate cancer in the lymph nodes before salvage lymph node dissection: a systematic review and meta-analysis. Prostate Cancer Prostatic Dis 2019;1:1-10.

25. Osmonov DK, Aksenov AV, Trick D, et al. Cancer-specific and overall survival in patients with recurrent prostate cancer who underwent salvage extended pelvic lymph node dissection. BMC Urol 2016;1:56.

26. Rigatti P, Suardi N, Briganti A, et al. Pelvic/retroperitoneal salvage lymph node dissection for patients treated with radical prostatectomy with biochemical recurrence and nodal recurrence detected by [11C] choline positron emission tomography/computed tomography. Eur Urol 2011;5:935-43.

27. Jilg CA, Rischke HC, Reske SN, et al. Salvage lymph node dissection with adjuvant radiotherapy for nodal recurrence of prostate cancer. J Urol 2012; 6:2190-7.

28. Suardi N, Gandaglia G, Gallina A, et al. Long-term outcomes of salvage lymph node dissection for clinically recurrent prostate cancer: results of a single-institution series with a minimum follow-up of 5 years. Eur Urol 2015;2:299-309.

29. Claeys T, Van Praet C, Lumen N, et al. Salvage pelvic lymph node dissection in recurrent prostate cancer: surgical and early oncological outcome. BioMed Res Int 2015;2015.

30. Tilki D, Mandel P, Seeliger F, et al. Salvage lymph node dissection for nodal recurrence of prostate cancer after radical prostatectomy. J Urol 2015;2:484-90.

31. Karnes RJ, Murphy CR, Bergstralh EJ, et al. Salvage lymph node dissection for prostate cancer nodal recurrence detected by $11 \mathrm{C}$-choline positron emission tomography/computerized tomography J Urol 2015;193:111-6.

32. Zattoni F, Nehra A, Murphy CR, et al. Mid-term outcomes following salvage lymph node dissection for prostate cancer nodal recurrence status post-radical prostatectomy. Eur Urol Foc 2016;5:522-31. 
33. Montorsi F, Gandaglia G, Fossati N, et al. Robot-assisted salvage lymph node dissection for clinically recurrent prostate cancer. Eur Urol 2017;3:432-8.

34. Herlemann A, Kretschmer A, Buchner A, et al. Salvage lymph node dissection after 68Ga-PSMA or 18F-FEC PET/CT for nodal recurrence in prostate cancer patients. Oncotarget 2017;48:84180.

35. Linxweiler J, Saar M, Al-Kailani Z, et al. Robotic salvage lymph node dissection for nodal-only recurrences after radical prostatectomy: perioperative and early oncological outcomes. Surg Oncol 2018;2:138-45.

36. Dietlein M, Kobe C, Kuhnert G, et al. Comparison of [18 F] DCFPyL and [68 Ga] GaPSMA-HBED-CC for PSMA-PET imaging in patients with relapsed prostate cancer. $\mathrm{Mol}$ Imaging and Biol 2015;4:575-84.

37. Hanske J, Ostholt J, Roghmann F, et al. Salvage lymph node dissection in hormone-naïve men: How effective is surgery?. Urol Oncol 2019;11:812-17.

38. Nam W, Choi SY, Yoo SJ, et al. Factors associated with testosterone recovery after androgen deprivation therapy in patients with prostate cancer. Investig Clin Urol 2018;1:18-24.

39. Bouchelouche K, Choyke PL. PSMA PET in prostate cancer-a step towards personalized medicine. Curr Opin Oncol 2016;3:216.

40. Afshar-Oromieh A, Avtzi E, Giesel FL, et al. The diagnostic value of PET/CT imaging with the 68 Ga-labelled PSMA ligand HBED-CC in the diagnosis of recurrent prostate cancer. Eur J Nucl Med Mol Imaging 2015;2:197-209.

41. Siriwardana A, Thompson J, van Leeuwen PJ, et al. Initial multicentre experience of 68Gallium-PSMA PET/CT guided robot-assisted salvage lymphadenectomy: acceptable safety profile but oncological benefit appears limited. BJUI 2017;5:673-81.

42. Maurer T, Gschwend JE, Eiber M. Prostate-specific membrane antigen-guided salvage lymph node dissection in recurrent prostate cancer: a novel technology to detect lymph node metastases. Curr Opin Urol 2018;2:191-6.

43. Rauscher I, Düwel C, Wirtz M, et al. Value of 111In-prostate-specific membrane antigen (PSMA)-radioguided surgery for salvage lymphadenectomy in recurrent prostate cancer: correlation with histopathology and clinical follow-up. BJUI 2017;1:40-7.

44. Ost P, Reynders D, Decaestecker K, et al. Surveillance or metastasis-directed therapy for oligometastatic prostate cancer recurrence: a prospective, randomized, multicenter phase II trial. J Clin Oncol 2018;5:446-456. 


\section{Figures and Tables}

Fig. 1. Waterfall plot of the percent change in prostate-specific antigen (PSA) from pre-surgical levels to nadir following salvage lymph node dissection.

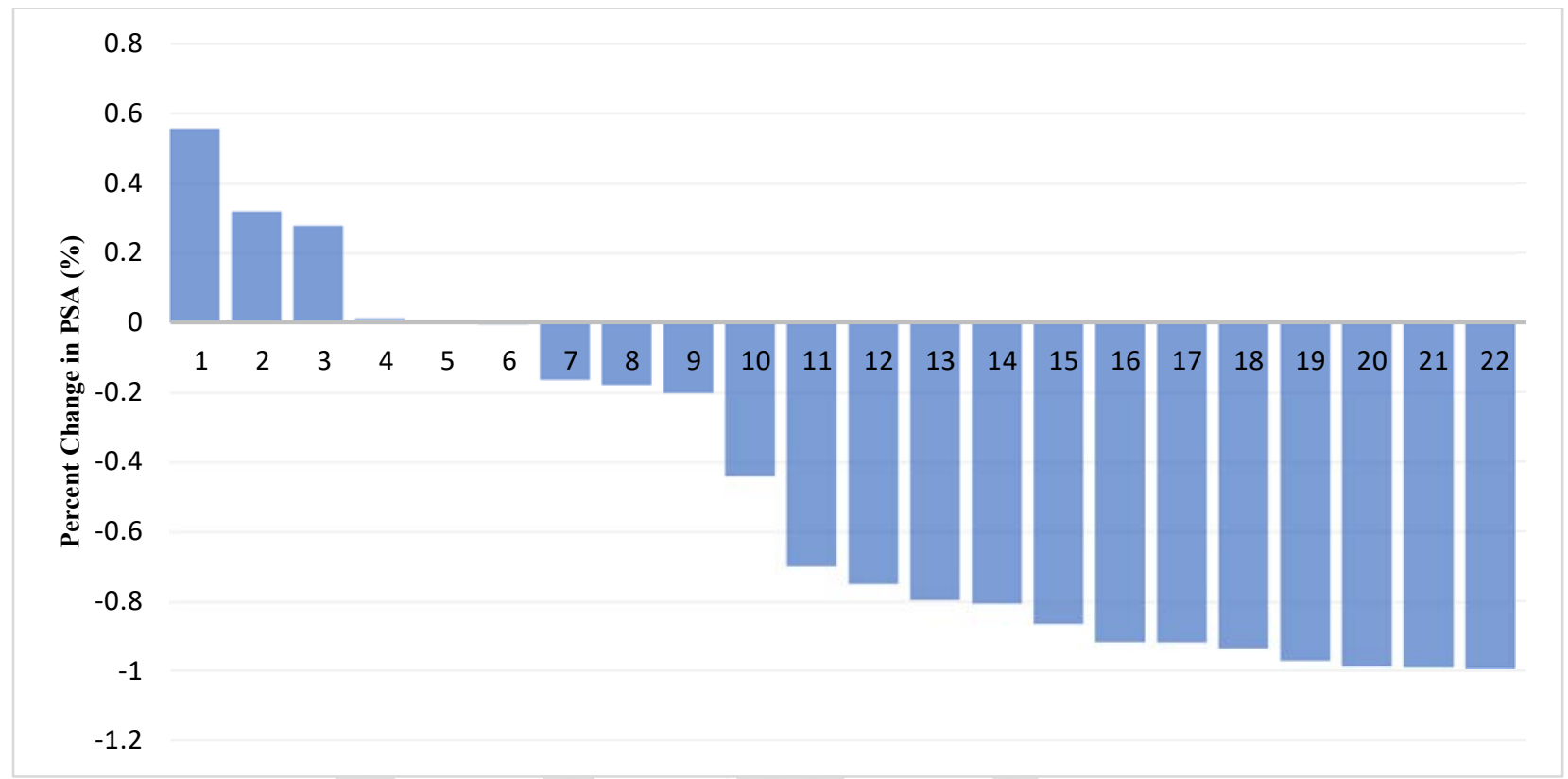


Fig. 2. Progression-free survival (PFS) of patients postoperatively (confidence interval [CI] of $95 \%)$.

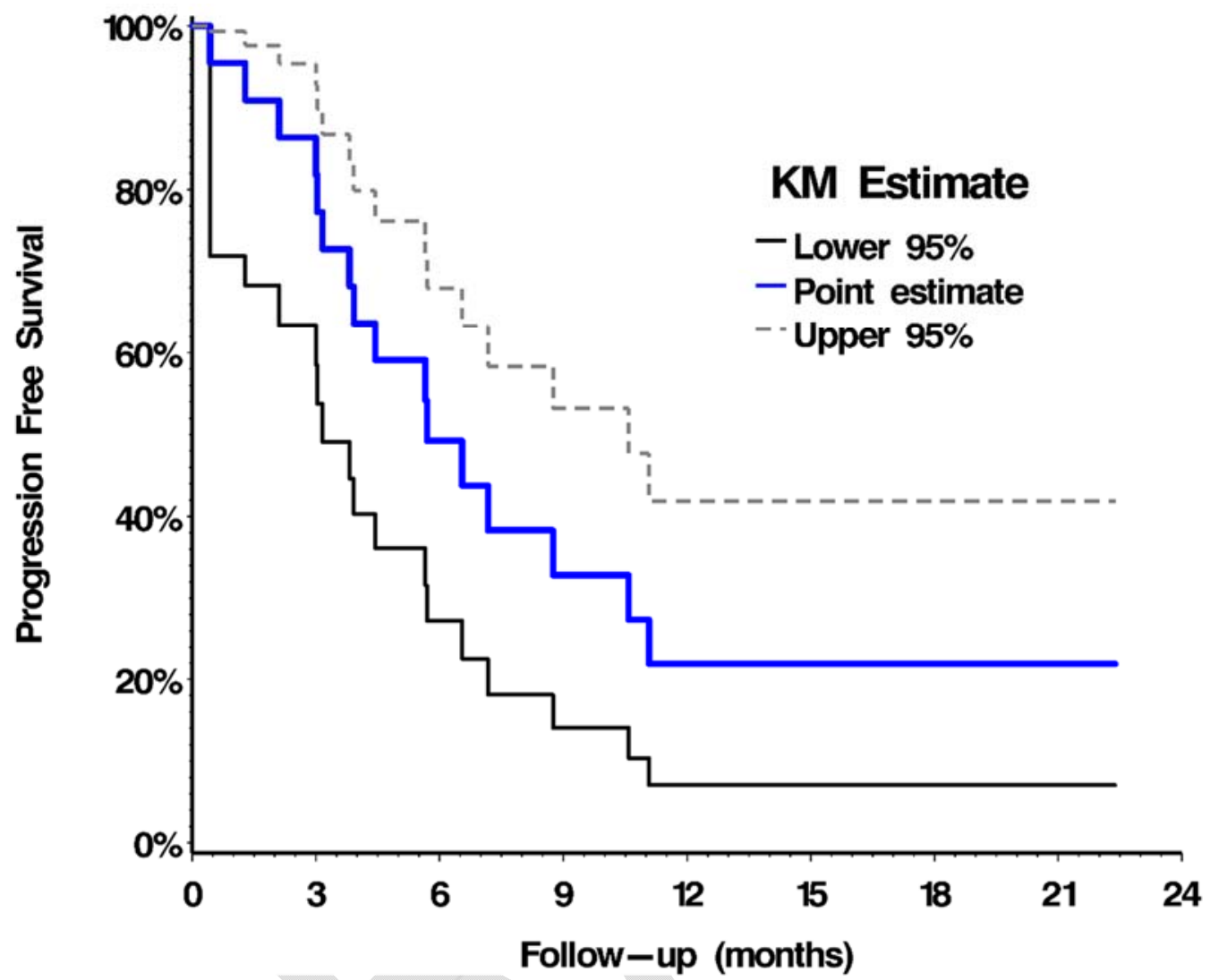

\begin{tabular}{|l|l|l|l|l|l|}
\hline \multirow{2}{*}{ Months } & \multirow{2}{*}{ PFS } & \multicolumn{2}{|l|}{$95 \%$ CI } & Patients \\
\cline { 3 - 6 } & & Lower & Upper & With event & at risk \\
\hline 3 & 0.8182 & 0.58535 & 0.92757 & 4 & 18 \\
\hline 6 & 0.4924 & 0.27256 & 0.67972 & 11 & 10 \\
\hline 9 & 0.3283 & 0.14044 & 0.53149 & 14 & 6 \\
\hline 12 & 0.2189 & 0.07035 & 0.41907 & 16 & 4 \\
\hline
\end{tabular}


Fig. 3. Metastasis-free survival of patients post-operatively (confidence interval [CI] of 95\%).

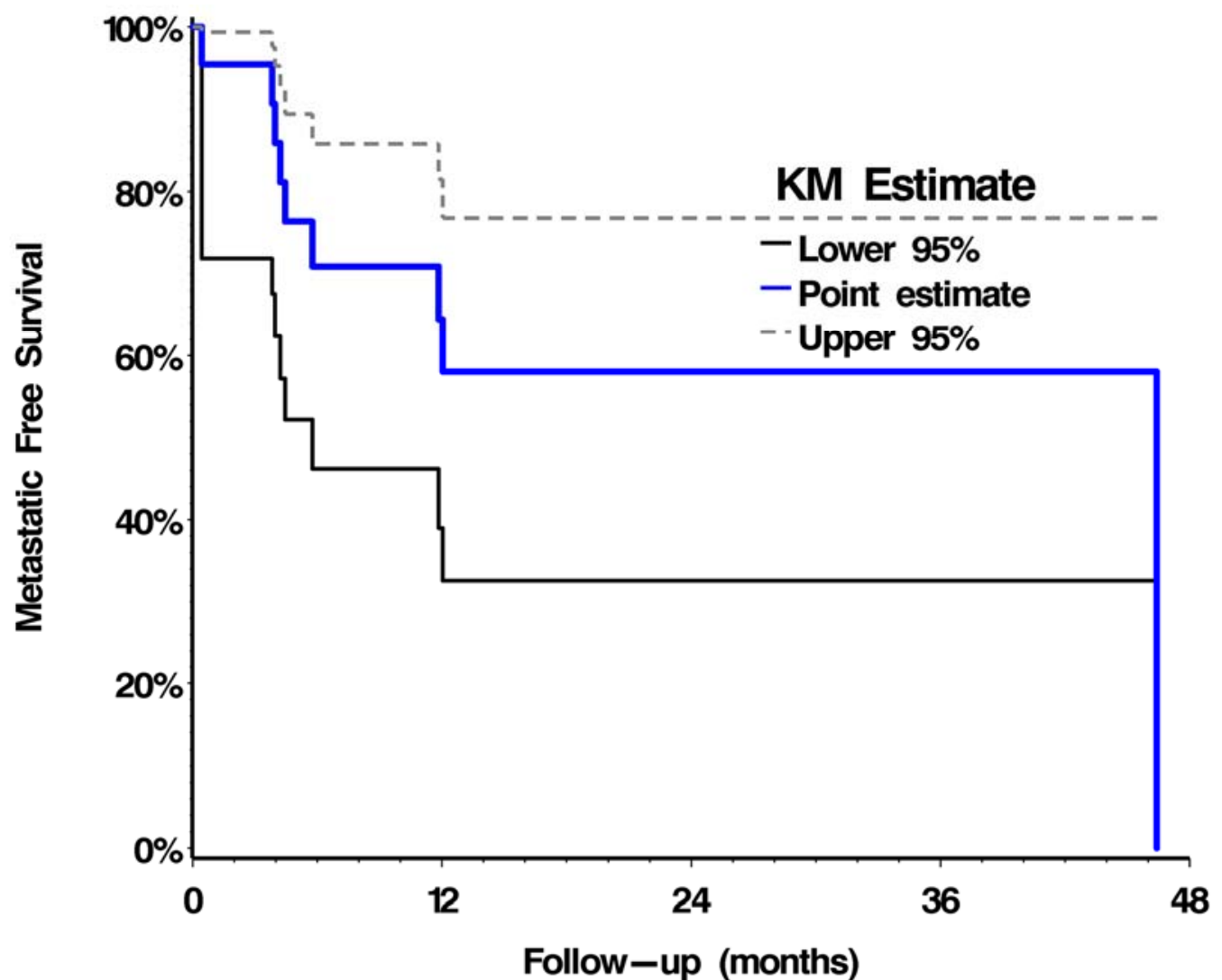

\begin{tabular}{|l|l|l|l|l|l|}
\hline \multirow{2}{*}{ Months } & MET-free & \multicolumn{2}{|l|}{$95 \%$ CI } & Patients \\
\cline { 3 - 6 } & survival & Lower & Upper & With event & at risk \\
\hline 3 & 0.9545 & 0.71870 & 0.99347 & 1 & 21 \\
\hline 6 & 0.7091 & 0.46191 & 0.85812 & 6 & 13 \\
\hline 9 & 0.7091 & 0.46191 & 0.85812 & 6 & 12 \\
\hline 12 & 0.6446 & 0.38981 & 0.81494 & 7 & 10 \\
\hline
\end{tabular}


Fig. 4. Androgen deprivation therapy free survival of patients postoperatively (confidence interval $[\mathrm{CI}]$ of $95 \%$ ).

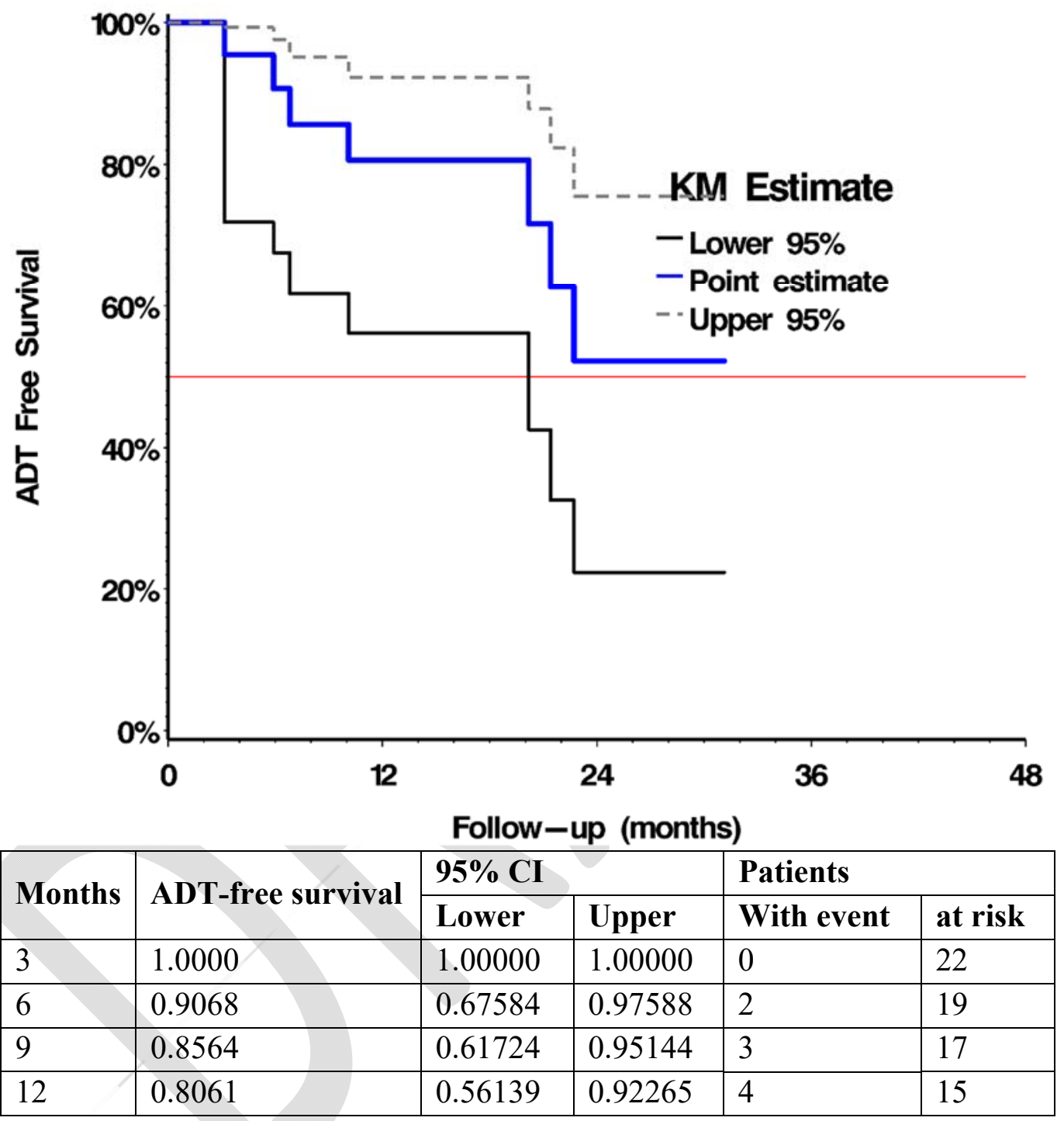




\begin{tabular}{|l|l|}
\hline \multicolumn{2}{|l|}{ Table 1. Baseline clinical and demographic factors } \\
\hline Variables & Value \\
\hline Median age at diagnosis, years (range) & $58(47-71)$ \\
\hline Median PSA at diagnosis, ng/ml (range) & $7.35(3.40-121.80)$ \\
\hline $\begin{array}{l}\text { Median BMI at primary treatment, } \mathrm{kg} / \mathrm{m}^{2} \\
\text { (range) }\end{array}$ & $29.2(21.9-41.5)$ \\
\hline Mode of primary treatment, $\mathrm{n}(\%)$ & \\
\hline $\begin{array}{l}\text { Radical prostatectomy with lymph node } \\
\text { dissection }\end{array}$ & $19(86.4 \%)$ \\
\hline Brachytherapy & $3(13.6 \%)$ \\
\hline Gleason score at primary treatment, $\mathrm{n}(\%)$ & \multicolumn{2}{|l|}{} \\
\hline 6 & $4(18.2 \%)$ \\
\hline 7 (Unspecified) & $1(4.55 \%)$ \\
\hline $7(3+4)$ & $11(50.0 \%)$ \\
\hline $7(4+3)$ & $6(27.3 \%)$ \\
\hline pT stage at primary treatment, $\mathrm{n}(\%)$ & $3(13.6 \%)$ \\
\hline pT1c & $3(13.6 \%)$ \\
\hline pT2a & $1(4.55 \%)$ \\
\hline pT2b & $1(4.55 \%)$ \\
\hline pT2c & $9(40.9 \%)$ \\
\hline pT3a & $3(13.6 \%)$ \\
\hline pT3b & $2(9.09 \%)$ \\
\hline N/A & $18(81.8 \%)$ \\
\hline pN stage at primary treatment, $\mathrm{n}(\%)$ & $1(4.55 \%)$ \\
\hline pN0 & $3(13.6 \%)$ \\
\hline pN1 & $7(36.8 \%)$ \\
\hline N/A & $12(63.2 \%)$ \\
\hline Surgical margins, $\mathrm{n}$ (\%) & $4(2-25)$ \\
\hline Positive & $18(94.7 \%)$ \\
\hline Negative & $1(5.26 \%)$ \\
\hline Median number of nodes removed (range) & $0.06(0.01-14.30)$ \\
\hline Number of positive nodes, $\mathrm{n}(\%)$ & $729(45-4026)$ \\
\hline 0 & $13(59.1 \%)$ \\
\hline 2 & $5(22.7 \%)$ \\
\hline $\begin{array}{l}\text { Median nadir PSA following primary } \\
\text { treatment (range) }\end{array}$ & \\
\hline $\begin{array}{l}\text { Median time to PSA rise following primary } \\
\text { treatment (range) }\end{array}$ & \\
\hline Mode of treatment following primary treatment \\
\hline Radiation therapy & \\
\hline ADT and radiation therapy & \\
\hline & \\
\hline
\end{tabular}




\begin{tabular}{|l|l|}
\hline \multicolumn{1}{|c|}{ None } & $4(18.2 \%)$ \\
\hline Duration of ADT use, days (range) & $294(183-1961)$ \\
\hline $\begin{array}{l}\text { Median time from prostate cancer diagnosis } \\
\text { to diagnosis of oligometastatic lesions on } \\
\text { PSMA PET/CT imaging }\end{array}$ & 5.80 \\
\hline
\end{tabular}

ADT: androgen deprivation therapy; BMI: body mass index; PSA: prostate-specific antigen; PSMA PET/CT: prostate-specific membrane antigen positron emission tomography/computed tomography.

\begin{tabular}{|c|c|}
\hline Imaging outcomes & Value \\
\hline \multicolumn{2}{|l|}{ Preoperative imaging } \\
\hline Median PSA level at PSMA PET scan (range) & $1.50(0.22-4.18)$ \\
\hline Median number of PSMA-Avid sites (range) & $2(1-5)$ \\
\hline \multicolumn{2}{|l|}{$\begin{array}{l}\text { Location of metastases on preoperative PSMA } \\
\text { PET/CT scan }\end{array}$} \\
\hline Pelvic nodes alone, $\mathrm{n}(\%)$ & $14(63.6 \%)$ \\
\hline Retroperitoneal nodes alone, $\mathrm{n}(\%)$ & $2(9.09 \%)$ \\
\hline Both pelvic and retroperitoneal nodes, $\mathrm{n}(\%)$ & $4(18.2 \%)$ \\
\hline Pelvic nodes + prostate bed, $\mathrm{n}(\%)$ & $2(9.09 \%)$ \\
\hline \multicolumn{2}{|l|}{ Findings on conventional imaging preoperatively } \\
\hline $\mathrm{CT}$ & $\begin{array}{l}4(18.2 \%) \\
\text { *Enlarged lymph nodes concordant with } \\
\text { PSMA PET/CT findings }\end{array}$ \\
\hline MRI & $0(0.00 \%)$ \\
\hline Bone scan & $0(0.00 \%)$ \\
\hline \multicolumn{2}{|l|}{ Postoperative lesion detection on imaging } \\
\hline Repeat PSMA, n (\%) & $5(22.7 \%)$ \\
\hline $\mathrm{CT}, \mathrm{n}(\%)$ & $4(18.2 \%)$ \\
\hline \multicolumn{2}{|l|}{ Location of relapse } \\
\hline Common iliac lymph node, n (\%) & $6(66.7 \%)$ \\
\hline Para-aortic lymph node, n (\%) & $2(22.2 \%)$ \\
\hline Aortocaval lymph node, $\mathrm{n}(\%)$ & $2(22.2 \%)$ \\
\hline Inguinal lymph node, $\mathrm{n}(\%)$ & $2(22.2 \%)$ \\
\hline Internal iliac lymph node, n (\%) & $1(11.1 \%)$ \\
\hline External iliac lymph node, n (\%) & $1(11.1 \%)$ \\
\hline Pre-sacral lymph node, n (\%) & $1(11.1 \%)$ \\
\hline Pelvic lymph node, $\mathrm{n}(\%)$ & $1(11.1 \%)$ \\
\hline Liver, $\mathrm{n}(\%)$ & $1(11.1 \%)$ \\
\hline \multicolumn{2}{|l|}{ Pathological outcomes } \\
\hline Median positive nodes removed (range) & $1.5(0-11)$ \\
\hline Median lymph node yield (range) & $10.5(2-42)$ \\
\hline
\end{tabular}




\begin{tabular}{|l|l|}
\hline Extranodal extension, $\mathrm{n}(\%)$ & $7(31.8 \%)$ \\
\hline $\begin{array}{l}\text { Positive margins following salvage therapy, } \mathrm{n} \\
(\%)\end{array}$ & $0(0.00 \%)$ \\
\hline
\end{tabular}

CT: computed tomography; MRI: magnetic resonance imaging; PET: positron emission tomography; PSA: prostate-specific antigen; PSMA: prostate-specific membrane antigen.

\begin{tabular}{|c|c|}
\hline Surgical outcome & Value \\
\hline \multicolumn{2}{|l|}{ Type of surgery } \\
\hline Pelvic lymph node dissection, $\mathrm{n}(\%)$ & $16(72.7 \%)$ \\
\hline Unilateral pelvic lymph node dissection, $\mathrm{n}(\%)$ & $12(54.6 \%)$ \\
\hline Bilateral pelvic lymph node dissection, $\mathrm{n}(\%)$ & $4(18.9 \%)$ \\
\hline $\begin{array}{l}\text { Retroperitoneal lymph node dissection alone, } \mathrm{n} \\
(\%)\end{array}$ & $2(9.09 \%)$ \\
\hline $\begin{array}{l}\text { Combination of procedures (PLND + } \\
\text { RPLND), } \mathrm{n}(\%)\end{array}$ & $4(18.9 \%)$ \\
\hline Salvage prostate procedures, $\mathrm{n}(\%)$, type & $1(4.55 \%)$, photodynamic therapy \\
\hline \multicolumn{2}{|l|}{ Surgical approach } \\
\hline Robotic procedure, $\mathrm{n}(\%)$ & $2(9.09 \%)$ \\
\hline Open procedure, $\mathrm{n}(\%)$ & $20(95.5 \%)$ \\
\hline \multicolumn{2}{|l|}{ PSA outcomes } \\
\hline Median preoperative PSA, ng/ml (range) & $1.72(0.22-10.7)$ \\
\hline $\begin{array}{l}\text { Median postoperative PSA nadir, ng/ml } \\
\text { (range) }\end{array}$ & $0.42(0.008-8.98)$ \\
\hline $\begin{array}{l}\text { Median time before PSA rise following } \\
\text { salvage surgery, days (range) }\end{array}$ & $185.5(39-339)$ \\
\hline \multicolumn{2}{|l|}{ Treatment following salvage surgery } \\
\hline ADT, $n(\%)$ & 7 \\
\hline Radiotherapy, n (\%) & 3 \\
\hline Chemotherapy, n (\%) & 1 \\
\hline \multicolumn{2}{|l|}{ Median time to subsequent treatment (range, months) } \\
\hline ADT & $10.1(3.16-22.7)$ \\
\hline Radiotherapy & $6.08(1.58-13.6)$ \\
\hline Chemotherapy & $5.69(\mathrm{~N} / \mathrm{A})$ \\
\hline
\end{tabular}

ADT: androgen deprivation therapy; PLND: pelvic lymph node dissection; PSA: prostatespecific antigen; RPLND: retroperitoneal lymph node dissection.

\begin{tabular}{|l|l|}
\hline \multicolumn{2}{|l|}{ Table 4. Surgical safety outcomes } \\
\hline Surgical outcomes & Value \\
\hline Median length of surgery, minutes (range) & $207.5(89.0-499.0)$ \\
\hline Median estimated blood loss, ml (range) & $100(50-2500)$ \\
\hline
\end{tabular}




\begin{tabular}{|l|l|}
\hline Blood transfusions, $\mathrm{n}(\%)$ & $1(4.55 \%)$ \\
\hline $\begin{array}{l}\text { Median postoperative length of stay, days } \\
\text { (range) }\end{array}$ & $2(1-9)$ \\
\hline Complication (Clavien-Dindo) & \\
\hline Grade I, $\mathrm{n}(\%)$ & $1(4.55 \%)$ \\
\hline Grade II, $\mathrm{n}(\%)$ & $1(4.55 \%)$ \\
\hline Grade IIIb, n (\%) & $2(9.09 \%)$ \\
\hline
\end{tabular}

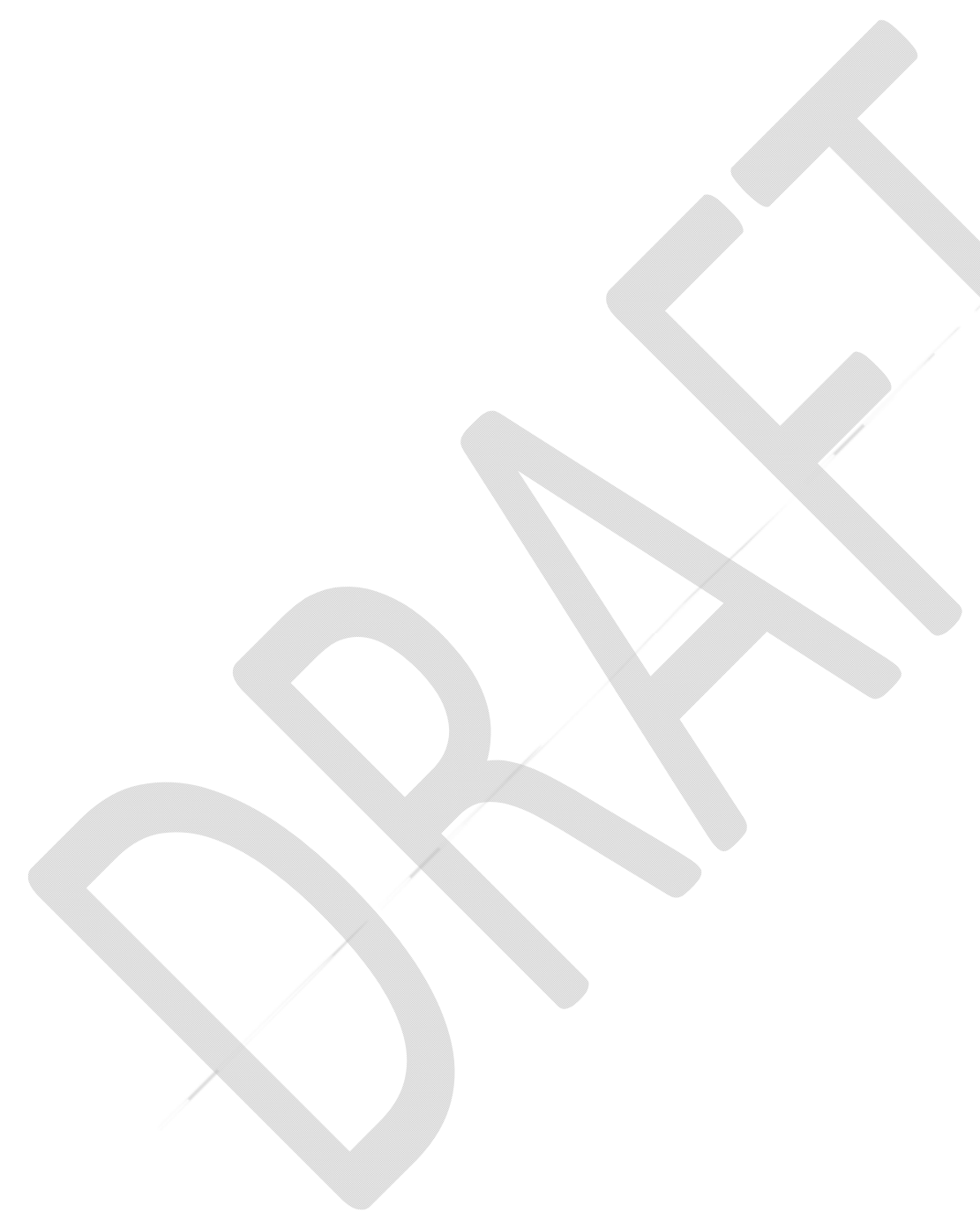

